\title{
Development, Validation and Application of a UHPLC-MS Method for the Quantification of Chios Mastic Gum Triterpenoids in Human Plasma\#
}

\author{
Authors \\ Petros Gkiouvetidis ${ }^{1}$, Apostolis Angelis ${ }^{1}$, Maria Halabalaki ${ }^{1}$

\section{Affiliations} \\ 1 Division of Pharmacognosy and Natural Products Chemis- \\ try, Department of Pharmacy, National and Kapodistrian \\ University of Athens, Panepistimiopolis Zografou, Athens, \\ Greece \\ 2 Department of Nutrition and Dietetics, School of Health \\ Sciences and Education, Harokopio University, Athens, \\ Greece
}

Vincent Brieudes ${ }^{1 *}$, Eleni V. Mikropoulou ${ }^{1 *}$, Errikos Kallergis ${ }^{1}$, Andriana C. Kaliora ${ }^{2}$, Efstathia Papada ${ }^{2}$,

\section{Key words}

Pistacia lentiscus var. Chia, Anacardiaceae, plasma, isomasticadienonic acid, mastic, masticadienonic acid, triterpenic acid

\section{received}

December 14, 2020

accepted after revision

February 25, 2021

published online

March 30, 2021

\section{Bibliography}

Planta Med 2021; 87: 1101-1109

DOI 10.1055/a-1408-9338

ISSN 0032-0943

(c) 2021. Thieme. All rights reserved.

Georg Thieme Verlag KG, Rüdigerstraße 14,

70469 Stuttgart, Germany

\section{Correspondence}

\section{Assistant Professor Maria Halabalaki}

Division of Pharmacognosy and Natural Products Chemistry, Department of Pharmacy, National and Kapodistrian University of Athens

Panepistimiopolis Zografou, 15771 Athens, Greece

Phone: + 302107274781 , Fax: + 302107274594

mariahal@pharm.uoa.gr
$\Theta$ Supplementary material is available under https://doi.org/10.1055/a-1408-9338

\section{ABSTRACT}

Chios mastic gum is the resinous secretion obtained from the barks of the shrub Pistacia lentiscus var. Chia, which is endemic to the Greek island of Chios. Since antiquity, Chios mastic gum has found several uses as a phytotherapeutic remedy, primarily for the treatment of gastrointestinal disorders while recently, Chios mastic gum was also recognized by EMA as an herbal medicinal product with specific indications. Chios mastic gum's biological properties are attributed to triterpenes which comprise the major chemical group (approx. $70 \%$ ) and notably isomasticadienonic acid and masticadienonic acid. However, due to their structural characteristics, the isolation thereof in high yield and purity is challenging and since they are not commercially available, pharmacological studies aiming to assess their biological properties are limited. In the present work, mastic's phytochemical investigation by UPLC-HRMS is followed by the isolation and characterization of isomasticadienonic acid and masticadienonic acid to be used as analytical standards for their accurate and reliable quantification in human plasma. A UHPLC-tQ-MS method that was developed and validated (in terms of specificity, linearity, limit of quantification, accuracy and precision), for the direct quantification of the targeted compounds in the low $\mathrm{ng} / \mathrm{mL}$ range of concentration, was subsequently implemented on plasma samples of healthy volunteers thus demonstrating its fitness for purpose. The results presented herein might provide insight to the understanding of this traditional natural product consumed notably for its anti-inflammatory, antioxidant and lipid lowering properties. Moreover, this method might serve as a starting point for any study aiming to monitor bioactive triterpenes in biological fluids. 


$\begin{array}{ll}\text { ABBREVIATIONS } \\ \text { ACN } & \text { acetonitrile } \\ \text { AF } & \text { mastic's acidic fraction } \\ \text { ANOVA } & \text { analysis of variance } \\ \text { APCI } & \text { atmospheric pressure chemical ionization } \\ \text { CD } & \text { Crohn's disease } \\ \text { CMG } & \text { Chios mastic gum } \\ \text { ESI } & \text { electrospray ionization } \\ \text { GT } & \text { glutamyl transferase } \\ \text { HDL } & \text { high density lipoprotein } \\ \text { IMLA } & \text { isomasticadienolic acid } \\ \text { IMNA } & \text { isomasticadienonic acid } \\ \text { IS } & \text { internal standard } \\ \text { LDL } & \text { low density lipoprotein } \\ \text { LOQ } & \text { limit of quantification } \\ \text { MLA } & \text { masticadienolic acid } \\ \text { MNA } & \text { masticadienonic acid } \\ \text { NF } & \text { mastic's neutral fraction } \\ \text { RDBeq } & \text { relative double bond equivalents } \\ \text { (R)SD } & \text { (relative) standard deviation } \\ \text { SFC } & \text { supercritical fluid chromatography } \\ \text { SGOT } & \text { serum glutamic oxaloacetic transaminase } \\ \text { SGPT } & \text { serum glutamic pyruvic transaminase } \\ \text { TMEWP } & \text { total mastic extract without polymer } \\ \text { tQ } & \text { triple-quadrupole mass analyzer } \\ & \end{array}$

\section{Introduction}

Chios mastic (mastiha or $\mathrm{CMG}$ ) is the resinous secretion obtained from the wounds of the trunk and branches of Pistacia lentiscus L. var. Chia, a shrub of the Anacardiaceae family. Even if $P$. lentiscus shrubs are widespread in the Mediterranean basin, the exclusive source of mastic resin is only the trees of the Chia variety growing on the Greek island of Chios [1]. Mastiha is strongly linked to the insular tradition of Chios, and systematic cultivations dominate mainly the south part of the island due to the particular pedoclimatic and microclimatic conditions [2]. CMG is a product with protected designated origin and since 2014, the traditional cultivation of mastic resin in Chios is recognized as intangible cultural heritage by UNESCO [3]. Traditional usage of mastic as a spice and a therapeutic agent dates back more than 2500 years. The ancient Greek physicians mentioned its properties and recommended its use especially for stomach and liver problems. Mastic's trade and value flourished during the Byzantine years and Medieval ages, when CMG occupied a special spot in folk medicine and, later on, in official Pharmacopoeias across Europe and Asia [4].

Several recent studies have attempted to investigate mastic's pharmacological potential. In fact, CMG has been found to possess anti-ulcer [5], anti-dyspepsia [6], as well as antifungal and antibacterial activities [7-9]. In 2015, an assessment of mastiha was initiated by the committee on Herbal Medicinal Products of the European Medicines Agency as a traditional herbal medicinal product with the following indications: treatment of mild dyspeptic disorders (indication 1) and symptomatic treatment of minor inflammations of the skin and as an aid in the healing of minor wounds (indication 2) $[10,11]$. Mastiha was also found to be effective against $H$. pylori [12] and to demonstrate positive action against CD [13] as well as have antiproliferative activity in colon and prostate models in vitro and in vivo [14-18]. Back in 1975, Abdel-Rahman et al. [19] investigated the antioxidant activity of mastic, showing it was comparable to that of butylated hydroxyanisol, the most used synthetic antioxidant in the food industry. Later, other studies confirmed the potent antioxidant character of mastic against, notably, the oxidation of animal and vegetal oils [20] as well as of human low-density lipoprotein [21]. Furthermore, it was also shown that mastic displays anti-inflammatory activity in human aortic endothelial cells [22]. Anti-inflammatory action of mastiha was also demonstrated in active CD patients who presented a significant reduction of the CD activity index and of plasma interleukin-6 and C-reactive protein levels after a 4-week administration [13].

Interestingly, following these findings, focus was given to its hypolipidemic activity [23]. A clinical trial on 133 human subjects over 50 years old receiving a different daily dose of mastiha over 18 and 12 months was conducted [24]. Results showed that the high-dosage group displayed a decrease in serum total cholesterol, LDL, total cholesterol/HDL ratio, lipoprotein, apolipoprotein A-1, apolipoprotein B (apoB/apoA-1 ratio did not change), SGOT, SGPT, and gamma-GT levels. Potential in vivo hepatoprotective and cardioprotective effects of mastic were recently studied further in both animals [25] and humans [26]. A pilot study was carried out with over 156 healthy human volunteers receiving daily doses of mastic under different forms over an 8-week period. It was found that a daily dose of $1 \mathrm{~g}$ of crude mastiha has a significant lowering effect on total cholesterol and glucose levels, with excellent tolerance and no detectable side effects, especially in overweight and obese individuals [26]. Meanwhile, many products containing mastic can be found on the market, e.g., flavoring, liquor, natural chewing gum, dentistry products, and dietary supplements and is used in traditional therapies (relief of gastralgia, protection against peptic ulcer) [4].

Responsible for the biological and pharmacological properties of mastiha is its unique composition. From a chemical point of view, it is a considerably complex mixture of approximately 120 constituents that have been reported so far. Triterpenoids comprise the major chemical group corresponding to the 67$70 \%$ of the total resins' weight. Terpenes have attracted particular attention from the scientific community and from the pharmaceutical industry for clinical studies and applications in the therapy of diseases due to their antioxidant [27] and anti-inflammatory properties, both associated with the biological activities reported in mastic [28]. It is worth noting that many triterpenoids of natural origin and/or their synthetic analogues are in the clinical trials stage [29].

In more detail, the main constituents of mastiha are the triterpenic acid isomers, namely, 24Z- IMNA, MNA, and oleanonic acid as well as 24Z-IMLA, MLA, and moronic acid [30,31]. In addition to the triterpenic acids, mastic resin also contains neutral triterpenes [32] and significant quantities of the polymer cis-1,4-poly- $\beta$-myrcene [33].

Nevertheless, despite the scientific interest of mastiha triterpenoids directly related to its biological properties and phytothera- 


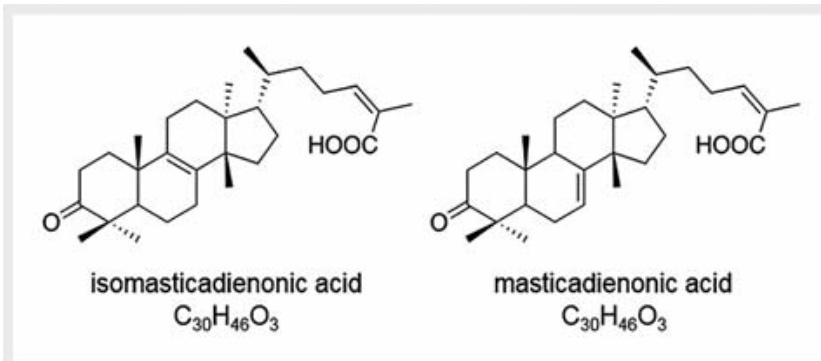

- Fig. 1 Structure of isolated compounds isomasticadienonic acid (IMNA) and masticadienonic acid (MNA).

peutic use, the studies investigating the activity profile thereof are noticeably limited. The same scarcity is also observed in the information related to pharmacokinetics and pharmacodynamics of mastiha components. It is worth mentioning that one of the reasons hindering such studies is the inherent complexity of the initial material and the coexistence of structurally close compounds as well as several isomers. Moreover, the presence of mastic's insoluble polymer greatly impedes sample handling and the overall phytochemical investigation of CMG's constituents. These facts lead to insufficient isolation procedures along with very low yields and purity, which inevitably results in the unavailability of reference compounds for further investigations [4]. Consequently, also similar is the lack of efficient and reliable analytical methods for the accurate identification of mastic triterpenoids and their quantitation, not only for quality control purposes (in resin) but also for the exploration of their pharmacological properties (in plasma, urine, tissues). However, this is a common obstacle in the field of bioactive natural products and their further development, since high yield and high purity are required to achieve meaningful results. To our knowledge, only two studies have been published dealing with this subject so far [34,35].

Thus, in the framework of a study on the bioavailability of mastic resin terpenes and postprandial effect on human antioxidant potential [36], the scope of the present work was to address the need for analytical tools for the direct quantification of mastic characteristic constituents IMNA and MNA. Initially, IMNA and MNA were isolated in pure form and high yield from CMG in order to be used as reference standards for the development and validation of an LC-MS methodology in human plasma, suggesting a complete workflow. The method was also validated according to $\mathrm{ICH}$ [37] and might serve as a valuable tool to any future study aiming to examine the pharmacological effect of CMG's constituents in human subjects.

\section{Results and Discussion}

As already mentioned, mastic resin is a very complex mixture of terpenes together with a natural polymer of poly- $\beta$-myrcene. Thus, the first and crucial step in CMG's extraction and phytochemical investigation was the removal of the insoluble polymer, which due to its gum-like formation, hinders sample handling and analysis. To this day, the most widely used method for polymer removal is the one that was first proposed by Barton and Seoane

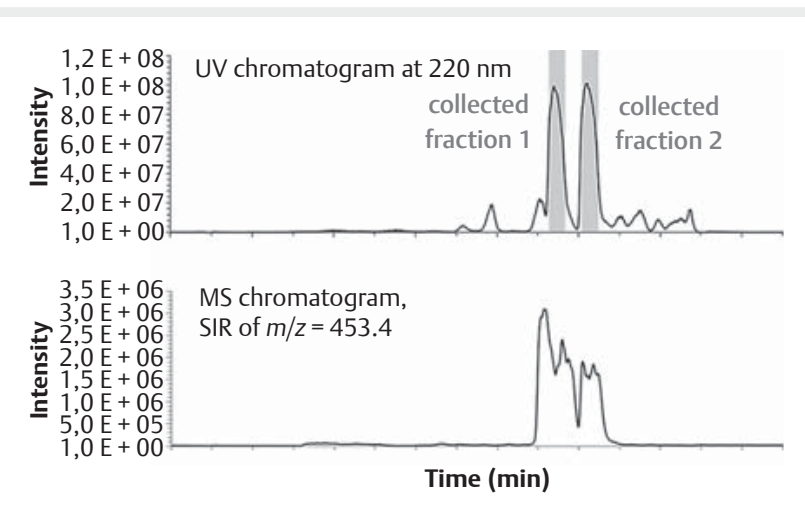

- Fig. 2 SFC-UV/MS chromatograms of the enriched extract from the acidic fraction (AF) of the total mastic extract without polymer (TMEWP) [upper, UV chromatogram at $220 \mathrm{~nm}$ and lower, ESI (-)-MS SIR chromatogram of $m / z=453.4$ ]; peaks at $R t=9.5$ and 10.2 minutes collected as fraction 1 and fraction 2 corresponds to isomasticadienonic acid (IMNA) and masticadienonic acid (MNA) respectively.

[30] and evolved by other research groups [12]. This protocol briefly involves the polymer's decantation in an appropriate organic solvent system and the consecutive partition of the supernatant between different $\mathrm{pH}$-altering biphasic systems affording the polymer, the total extract without polymer, the neutral terpenoids, and the acidic terpenoids into four separate fractions [12]. In the present study, $76 \mathrm{~g}$ of crude mastic powder were used as the raw material and after polymer decantation and filtering, $66.8 \mathrm{~g}$ of TMEWP were collected, $5 \mathrm{~g}$ of which were kept for further analysis. After partitioning, $15.2 \mathrm{~g}$ of NF and $1.7 \mathrm{~g}$ of AF were obtained. Profiling and characterization of terpenes took place afterwards to all three derived extracts (TMEWP, AF, NF) by UPLCHRMS using an Orbitrap analyzer. Due to the relatively low polarity of the terpenes of NF, apart from the ESI source, APCI was also employed (Fig. 1S, Supporting Information). Based on previous studies of our group [25,38], TMEWP and AF profiles are dominated by the presence of tetracyclic and pentacyclic triterpenes, and, most notably, the isomers moronic, oleanonic, MNA, and IMNA with the two last being tirucallane derivatives ( $\triangleright$ Fig. 1). All four compounds were detected at $\mathrm{m} / \mathrm{z}$ of $453.3376\left(\mathrm{C}_{30} \mathrm{H}_{45} \mathrm{O}_{3}\right.$, RDBeq. 8.5) and eluted in the abovementioned order (Fig. 1S, Supporting Information). The profile is complemented by other triterpenic acids, such as the isomers MLA and IMLA, at m/z 455.3533 $\left(\mathrm{C}_{30} \mathrm{H}_{47} \mathrm{O}_{3}\right.$, RDBeq. 7.5), eluted at 12.72 and $13.26 \mathrm{~min}$, which comprise the respective alcohols of MNA and IMNA.

Since the current study focuses on the characteristic triterpenic acids of mastic and their quantitation in plasma, the AF was forwarded for separation, isolation, and purification. Thus, semipreparative SFC-UV/MS using a chiral stationary phase and $\mathrm{MeOH}$ as the cosolvent was used for this purpose, an approach which has been previously proposed, with minor modifications [39]. As shown in - Fig. 2, this method offered a satisfactory separation between the two targeted compounds, which enabled their collection and isolation in high purity. Interestingly, the difference in response factors between UV and MS detection can be easily no- 
ticed. In the latter, a minor compound of the extract, i.e., peak eluted at $\mathrm{Rt}=9.0 \mathrm{~min}$, displayed an intensity greater than those of the target compounds. This illustrates the necessity of using relevant analytical standards for the implementation of MS-based quantitative methodologies for the measurement of mastic gum triterpenes. A total of $50 \mathrm{mg}$ of mastic's AF was processed. Next, the collected fractions were merged and $\mathrm{MeOH}$ was evaporated. The remaining quantities were $18.7 \mathrm{mg}$ for fraction 1 and $20.5 \mathrm{mg}$ for fraction 2, which corresponds IMNA and MNA, respectively. Both fractions were transferred for further analysis, including NMR and HRMS for structure verification and purity evaluation to be used as reference standards, with a purity of $>95 \%$ for IMNA and $>99 \%$ for MNA (Figs. 2S and 3S, Supporting Information).

Reference compounds of the analytical quality required is the most common issue that analytical chemists working in the field of natural products are facing when developing methodologies for the characterization of phytoconstituents in extracts or biological fluids. The most common way to overcome this issue is to use commercially available analytical standards of the same chemical family. The quantification of cinnamic acids as caffeic acid equivalents or polyphenols as gallic acid equivalents are well-known examples [40]. This strategy is very efficient when performing UV detectionbased analysis, although it is limited when seeking to reach a lower LOQ implementing MS detection-based analysis. Indeed, ionization efficiency and matrix effects can possibly lead to a great difference in the response factor even for compounds that are structurally close. In that case, isolation of the targeted compounds appears to be the best option, even though it might be a challenging task, especially in regard to complex mixtures and structurally close compounds as in the case of triterpenic acids of mastic.

The next step in the experimental process was the development and validation of the analytical method for the quantitation of MNA and IMNA in plasma. To satisfy the sensitivity aspects of the analysis, a UHPLC system hyphenated to tQ was employed. Due to the particular chemical nature of the analytes, a pseudoMRM (453.4 > 453.4) method was used. As illustrated in > Fig. 3 , where the reference compounds MNA (10.57 min) and IMNA (10.95 min) are compared with TMEWP, there are no coeluting compounds. The relative retention time of MNA and IMNA standards in relation to the TMEWP were 1.05 and 1.03 , respectively, and the resolution between compounds was 1.1 , thus demonstrating the effectiveness of the separation. It is useful to note that TMEWP displayed two other peaks eluting at 7.15 and 7.55 min identified as moronic acid and oleanonic acid, both isomers of MNA and IMNA [38]. Moreover, 3-O-acetyl ursolic acid was selected as an IS, demonstrating close structural features with MNA and IMNA (pseudo-molecular ion at $\mathrm{m} / \mathrm{z}$ 497.3634, $\left.\mathrm{C}_{32} \mathrm{H}_{49} \mathrm{O}_{4}, \Delta \mathrm{m}<0.5 \mathrm{ppm}\right)$. The IS was derived after acetylation of ursolic acid. Finally, the unequivocal assessment of the targeted compounds in the presence of the components that may be expected to be present was demonstrated.

A short-term stability study was initially conducted in order to demonstrate the extent to which the analyte's response is stable in defined conditions, so as to guarantee the reliability of the analytical process. Stability of MNA and IMNA was evaluated by the recovery of the response (ratio of area analyte/IS) of standard solutions at different time points with that of zero time. Stability
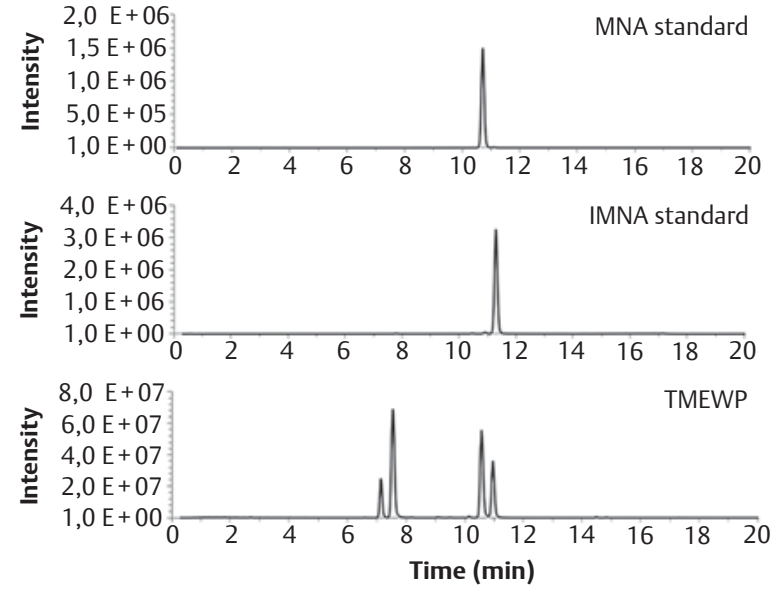

- Fig. 3 Pseudo-MRM (453.4 > 453.4) extracted ion chromatograms of masticadienonic acid (MNA) and isomasticadienonic acid (IMNA) standard solutions and of the total mastic extract without polymer (TMEWP) in ESI(-).

conditions were similar to those of the storage conditions of the samples to be further analyzed, i.e., $10^{\circ} \mathrm{C}$. Three independent replicates of standard solutions were freshly prepared in $\mathrm{MeOH}$ at three different concentrations $(20,100,300 \mathrm{ng} / \mathrm{mL}, \mathrm{n}=9)$ and were analyzed on 5 different days (days 1, 2, 3, 4, 5, and 7). The average recoveries of the replicate samples for every time point are displayed in $\mathbf{F i g . ~} 4$. At the low level of concentration (20 ng/ $\mathrm{mL}$ ), recovery for the IMNA standard was of $100.65 \%$ after 7 days and of $100.33 \%$ for MNA, while respective RSDs were 6.11 and $4.70 \%$. At $100 \mathrm{ng} / \mathrm{mL}$, the IMNA standard displayed recoveries ranging from 100.09 to $106.40 \%$ with respective RSDs of 5.73 and $1.16 \%$ over the investigated period. In the same conditions, MNA standard minimum and maximum recoveries were 99.43 and $100.56 \%$ with an RSD of 1.58 and $4.99 \%$ respectively. Similar results were observed for both compounds at $300 \mathrm{ng} / \mathrm{mL}$, with the average recovery for all investigated time points calculated at $102.27 \%$ for IMNA and $\mathbf{1 0 0 . 3 6 \%}$ for MNA. Corresponding RSDs were 4.63 and of $5.06 \%$, respectively. Finally, considering all analyzed samples and time points $(n=54)$, both solutions of IMNA and $\mathrm{MNA}$ in $\mathrm{MeOH}$ are stable during a period of at least 7 days at $10^{\circ} \mathrm{C}$, reaching a maximum acceptable deviation of signal response of $10 \%$. Consequently, all quantitative analysis took place within the characterized stability period.

Calibration curves were constructed as a function of area ratio (target compound/IS) against the concentration ratio (target compound/IS) for the 15 levels of concentration. Each level was randomly injected three times during the analytical sequence in-between all samples to be analyzed. Calibration curves for both targeted compounds in solvent are presented in Fig. 4S, Supporting Information. Coefficients of determination $\left(\mathrm{R}^{2}\right)$ were 0.9995 for MNA and 0.9966 for IMNA. Moreover, a statistical evaluation of model linearity by ANOVA was performed. First, homogeneity of variances between each concentration level was verified using Cochran's C test with a significance level $(\alpha)$ of 0.05 . In these condi- 
tions ( 15 series of 3 data points each), the upper limit critical value was 0.33 . The maximum observed $C$ value was of 0.24 for MNA and 0.30 for IMNA, therefore, variances can be considered homogeneous for both compounds, hence repeatability is stable in the calibration model range. Then, F-tests were performed at the $\alpha$ level of 0.05 to compare variances of modelized values against residuals. Calibration models were shown to be significant for both compounds. Indeed, the explained data's F values (mean square of modelized values/mean square of residuals) were greatly superior to $\mathrm{F}_{5 \%}$, which implies that the tested variances are not equals; the calibration model explains the data. Also, F values of "nonlinearity" (mean square of residuals/mean square of repeatability residues) were inferior to $\mathrm{F}_{5 \%}$; linearity of the models is verified, and the defined calibration models are suitable. ANOVA tables for both compounds are detailed in Table 1S, Supporting Information.

LOQ is described as "the lowest amount of analyte in a sample that can be determined with acceptable precision and accuracy under the stated experimental conditions" [37], and therefore it should belong to a range of concentrations for which the linearity of the concentration against the measured signal has been demonstrated. Hence, the first level of the calibration curve for each analyte was defined as the instrumental LOQ. Taking into account the dilution factor from the preparation of plasma samples, the method LOQ was determined to be in plasma $6 \mathrm{ng} / \mathrm{mL}$ for IMNA and $1 \mathrm{ng} / \mathrm{mL}$ for MNA. Consequently, three independent pooled samples of control plasma $(t=0)$ were spiked accordingly to verify method performance at the LOQ. At this level, the RSD of the signal response was of $12 \%$ for IMNA and $5 \%$ for MNA and the difference of the average signal response with this of a standard solution at the same concentration was $+27 \%$ and $-8 \%$ for IMNA and MNA, respectively.

Accuracy of an analytical method can be defined as the closeness of agreement between the measured value obtained by implementing this method and the true value [37]. This definition corresponds to what is designated as "trueness" in the International Vocabulary of Metrology and in ISO documents [41]. In the present work, the accuracy was evaluated by the preparation and analysis of three plasma samples artificially prepared so as to contain IMNA and MNA at low $(20 \mathrm{ng} / \mathrm{mL})$, medium $(100 \mathrm{ng} / \mathrm{mL})$, and high $(300 \mathrm{ng} / \mathrm{mL}$ ) levels of concentration. As described in the experimental section, the blank matrix consisting of pooled plasma samples of the zero time (pre-ingestion) were spiked accordingly and analyzed, following the whole analytical procedure including sample preparation. The experiment was then repeated after 1 week. The average measurement error in intermediate precision conditions ( $n=6,2$ days $\times 3$ independent replicates), that is to say the difference between the measured and the theoretical concentrations of the spiked samples, was determined for each investigated level of concentration.

Precision of an analytical method can be defined as "the closeness of agreement (degree of scatter) between a series of measurements" [37]. In the present study, precision of the method is expressed as the SD of the measurement bias of six independent samples analyzed in intermediate precision conditions. Results are presented in $\mathbf{F i g . ~ 5 . ~ A t ~ t h e ~ l o w ~ l e v e l ~ o f ~ c o n c e n t r a t i o n ~}(20 \mathrm{ng} /$ $\mathrm{mL}$ ), the measurement error of the IMNA standard was -6.88 and $-1.53 \%$ for MNA, while associated SDs were 6.86 and of $6.09 \%$ respectively. At $100 \mathrm{ng} / \mathrm{mL}$, the difference between the

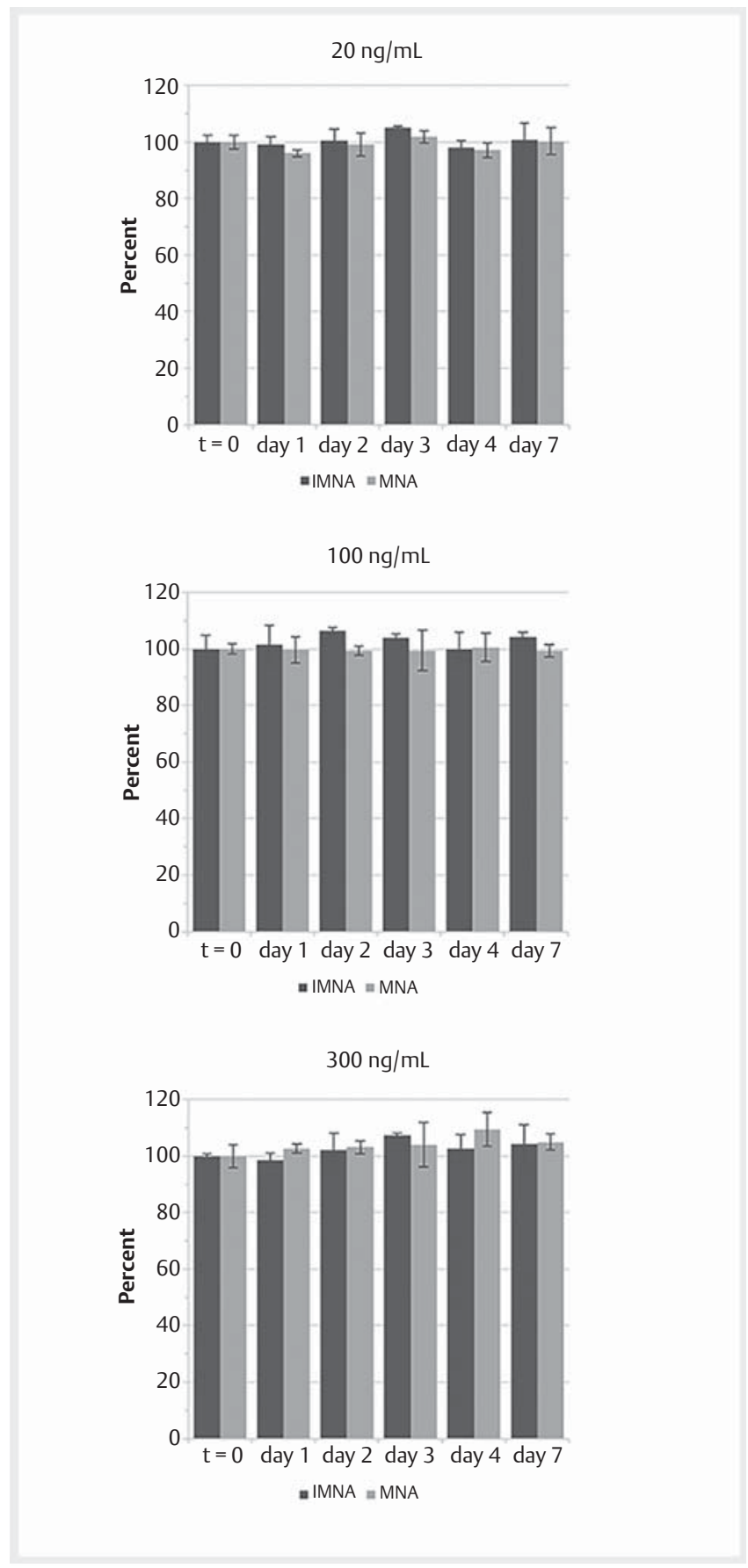

- Fig. 4 Seven-day stability of standard solutions at low, medium, and high concentrations (i.e., 20,100 , and $300 \mathrm{ng} / \mathrm{mL}$ ) kept at $10^{\circ} \mathrm{C}$ (error bars represent the relative SD between three independent replicates).

measured and theoretical concentrations was $-1.25 \%$ for IMNA and $1.35 \%$ for MNA. At this level, corresponding SDs of the measurement error were 4.26 and $4.00 \%$, respectively. Finally, at the high level of concentration $(300 \mathrm{ng} / \mathrm{mL})$, both analytes presented a slight negative measurement bias, which was $-7.69 \%$ for IMNA and $-7.45 \%$ for MNA, with associated SDs calculated at 6.47 and $4.90 \%$, respectively. Considering all analyzed samples, accuracy 


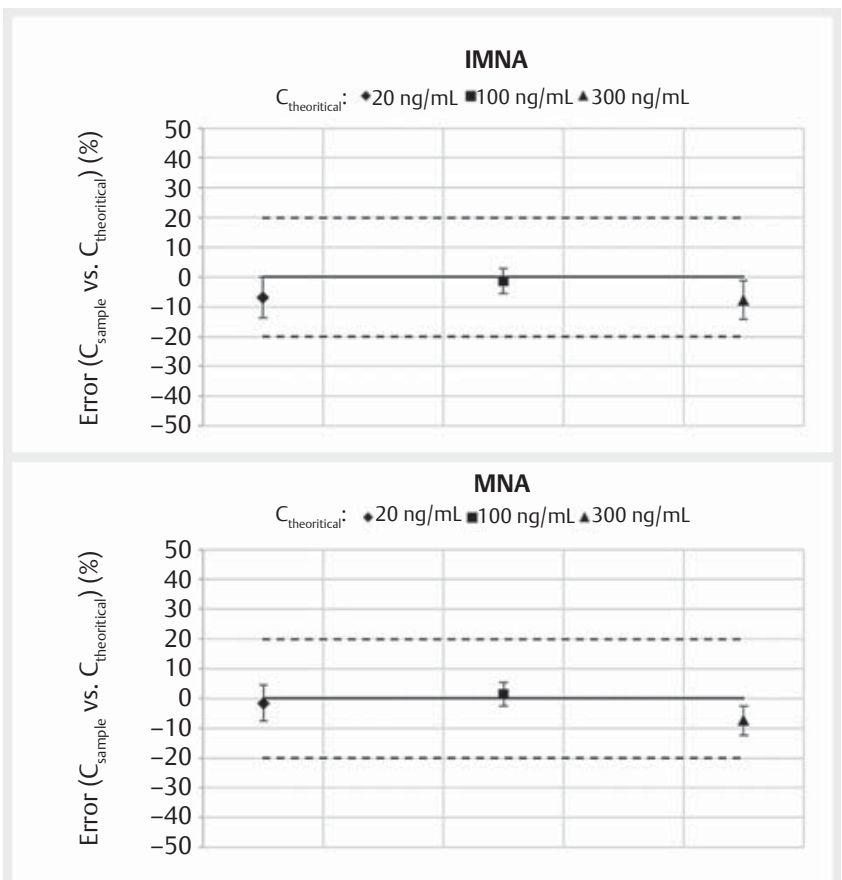

- Fig. 5 Measurement error of blank matrix (pooled plasma samples $\mathrm{t}=0$ ) spiked at low, medium, and high concentrations (i.e., 20, 100 , and $300 \mathrm{ng} / \mathrm{mL}$ ) analyzed in intermediate precision conditions ( $n=6: 2$ days $\times 3$ independent replicates, error bars represent the SD, dotted lines represent a maximum acceptable deviation 20\%).

and precision of the present method were demonstrated for both targeted compounds within a maximum acceptable deviation of $20 \%$ as shown in > Fig. 5 .

After validation of the initial performances, the herein method was implemented for the measurements of IMNA and MNA in plasma samples of 17 healthy male adults after oral administration of $10 \mathrm{~g}$ of CMG powder dispersed in water [36]. Suitability of the analytical system was checked by investigation of the internal quality control samples. None of the targeted compounds were detected in the procedural blank nor in the injection blanks, which were performed every 10 injections. Pooled QC samples were injected 5 times in the beginning, 5 times at the end of the sequence, and every 40 injections in-between. The RSD of the MNA signal response in the QC samples was 6.24 and $11.87 \%$ for IMNA. These performances are in accordance with the those demonstrated during the method validation. The MNA and IMNA average concentrations in QC plasma samples were $19.10 \mathrm{ng} / \mathrm{mL}$ and $31.57 \mathrm{ng} / \mathrm{mL}$, respectively. Plasma kinetic parameters are discussed and presented elsewhere [36] and will not be detailed here. Briefly, at zero time, both compounds were found to be inferior to the LOQ. The target compounds plasmatic concentrations then reached a maximum in the range of $30-767 \mathrm{ng} / \mathrm{mL}$ for MNA and $68-1381 \mathrm{ng} / \mathrm{mL}$ for IMNA before decreasing to a low $\mathrm{ng} / \mathrm{mL}$ after $24 \mathrm{~h}$. Maximum plasmatic concentrations were generally observed between 2 and $4 \mathrm{~h}$ after intake, even though this could vary depending on subjects.

To conclude, an analytical method using an LC-tQ platform was fully validated in order to establish for the first time reliable quan- titative data on the bioavailability of active terpenic acids from CMG in human plasma. Moreover, a complete workflow was designed from the purification of the compounds of interest, MNA and IMNA, in the required purity and yield, to a full validation of a method for their quantitation in human samples as well as its application to real samples. A semipreparative SFC-UV/MS apparatus could serve as a method of choice for terpenoids in complex mixtures offering high purity (dual detection), high yield, as well as speed due to the high degree of automation. Furthermore, the methods described herein can be used as a valuable tool in future pharmacological evaluations of CMG's constituents, since the detection and quantification of mastic's bioactive triterpenic acids in plasma might provide further insight on the dose/response relationship of mastic's supplementation.

\section{Materials and Methods}

\section{Chemicals and reagents}

For mastic's extraction, all solvents were of analytical grade and purchased from Sigma-Aldrich. $\mathrm{HCl}(37 \%)$ and $\mathrm{Na}_{2} \mathrm{CO}_{3}$ (99.5\%) were also purchased from Sigma. Based on previous works, 3-Oacetyl ursolic acid was used as an IS [34]. It was prepared by acetylation of commercial ursolic acid ( $p \geq 98.5 \%)$ with pyridine in diethyl ether, all acquired from Sigma-Aldrich. Food grade $\mathrm{CO}_{2}$ was obtained from Revival A.E. HPLC and LC-MS grade solvents were purchased from Merck Chemicals and high-purity water was provided by a Millipore Direct-Q 3 UV purification system. Optima LC-MS grade formic acid was obtained from Thermo Fisher Scientific and NMR solvents were purchased from Eurisotop.

\section{Chios mastic gum extraction}

CMG was kindly provided by the Chios Mastiha Growers Association. AF was obtained as previously described [12]. In brief, $76 \mathrm{~g}$ of crude mastic powder was dissolved in ethyl acetate/methanol $1: 3(600 \mathrm{~mL}$ in total). After 2 days at room temperature, the insoluble and decanted polymer was removed and the solution was filtered and condensed, giving a total of $66.8 \mathrm{~g}$ of TMEWP. TMEWP was thrice partitioned in a separatory funnel between aqueous $5 \%$ $\mathrm{Na}_{2} \mathrm{CO}_{3}$ and ether ( $1 \mathrm{~L}$ of each for all repetitions), which afforded $15.2 \mathrm{~g}$ of NF in the organic phase. The aqueous phase ( $3 \mathrm{~L}$ in total) was subsequently acidified with $1 \mathrm{~N} \mathrm{HCl}$. The acidic solution was reextracted with $1 \mathrm{~L}$ of ether, and the organic phase afforded $1.7 \mathrm{~g}$ of AF. All solvent evaporations were performed using a rotary evaporator (Buchi) with a water bath at $40^{\circ} \mathrm{C}$.

\section{Isolation of isomasticadienonic acid and masticadienonic acid as analytical standards}

MNA and IMNA were isolated by SFC instrumentation using a previously described method with some modifications [39]. In brief, instrumentation consisted of a Waters Prep 15 SFC Purification System coupled with a SQ Detector 2 mass spectrometer. The mass analyzer was interfaced with a Z-spray ion source operated in ESI negative. A ChiralPak IC column $(5 \mu \mathrm{m}, 4.6 \times 250 \mathrm{~mm})$ was used for the fractionation of the AF. The mobile phase was supercritical $\mathrm{CO}_{2}$ with $\mathrm{MeOH}$ as an organic modifier. Chromatographic flow rate was set at $5 \mathrm{~mL} / \mathrm{min}$. Gradient elution started at $5 \%$ co- 
solvent for 2 min and increased to $30 \%$ in 10 min before going back to the initial condition in $30 \mathrm{~s}$ for a 2.5 -min equilibration step. MS detection was carried out by simultaneously using an SIR function monitoring $\mathrm{m} / \mathrm{z}=453.40$ as well as a full scan function with a mass range of 200 to $800 \mathrm{amu}$.

\section{Characterization of mastic's fractions and the compounds used as analytical standards}

TMEWP, AF, and NF were analyzed by UPLC-HRMS. Liquid chromatography analysis was performed on an Acquity UPLC System. Detection was performed on a Thermo LTQ-Orbitrap XL hybrid mass spectrometer equipped with an ESI and an APCI source. Ten $\mu \mathrm{L}$ of each fraction at $100 \mu \mathrm{g} / \mathrm{mL}$ were injected into the system. Separation was achieved on a Supelco Ascentis C18 column $(15 \mathrm{~cm} \times 2.1 \mathrm{~mm}, 3 \mu \mathrm{m})$ using a water gradient containing $0.1 \%$ $(\mathrm{v} / \mathrm{v})$ formic acid (A) and ACN (B). Elution started at $5 \% \mathrm{~B}$, which increased to $70 \% \mathrm{~B}$ in $3 \mathrm{~min}$, reaching $100 \% \mathrm{~B}$ in a total of $20 \mathrm{~min}$. These conditions were maintained for $4 \mathrm{~min}$ before returning to initial conditions in $1 \mathrm{~min}$ for a 3 -min reequilibration (28 min in total). The column was maintained at $40^{\circ} \mathrm{C}$ and the flow rate was set to $0.4 \mathrm{~mL} / \mathrm{min}$. HRMS data were acquired in the negative mode in the full scan $\mathrm{m} / \mathrm{z}$ range of $115-1000$ with a resolution of 30000 . Data-dependent acquisition was simultaneously performed using a CID value of $35 \%$ and a mass resolution of 7500. For the ESI source, the capillary temperature was set to $350^{\circ} \mathrm{C}$ and the source voltage was $2.7 \mathrm{kV}$. Tube lens and capillary voltage were respectively tuned at $-100 \mathrm{~V}$ and $-30 \mathrm{~V}$. Nitrogen was used as the sheath gas (40 au) and auxiliary gas (10 au). For the APCl source, the capillary temperature was set to $200^{\circ} \mathrm{C}$, the $\mathrm{APCl}$ vaporizer temperature was set to $400^{\circ} \mathrm{C}$, and the source voltage was $6 \mathrm{kV}$. Tube lens and capillary voltage were respectively tuned at -125 and $-30 \mathrm{~V}$. Nitrogen was used as the sheath gas (70 au) and auxiliary gas (20 au). Spectral interpretation was performed using Xcalibur (Version 2.2) software.

Isolated compounds and the IS were characterized by HRMS and NMR. Direct infusion experiments were performed on an LTQ-Orbitrap XL hybrid mass spectrometer equipped with an ESI source. MS data were acquired in the negative mode in the full scan $\mathrm{m} / \mathrm{z}$ range of 100-1000 with a resolution of 30000. 1D and 2D nuclear magnetic resonance spectra $\left({ }^{1} \mathrm{H},{ }^{13} \mathrm{C}, \mathrm{COSY}, \mathrm{COSY}-\mathrm{LR}\right.$, HSQC-DEPT, HMBC) were acquired on a Bruker Avance $600 \mathrm{MHz}$ spectrometer using $\mathrm{CDCl}_{3}$ as the solvent. Chemical shifts $(\delta)$ are expressed in ppm with reference to the solvent signals $\left(\delta_{H} 7.26\right)$ $\left.\delta_{C} 77.0\right)$.

\section{Recruitment of the healthy volunteers and dosage information}

Apparently healthy men, aged 20-40 years old, were invited for screening (ClinicalTrials.gov Identifier: NCT02847117). The study protocol was reviewed and approved by the Harokopio University Ethics Committee (49/29-10-2015). It was conducted according to the principles of the Declaration of Helsinki and followed the Principles of Good Clinical Practice. Enrollment was based on certain inclusion and exclusion criteria. More specifically, a BMI $>26 \mathrm{~kg} / \mathrm{m}^{2}$, history of alcohol or drug abuse, medication, vitamin or inorganic supplements intake as well as following a vegan or macrobiotic diet were considered exclusion criteria. A subject pre- senting gastrointestinal diseases such as IBD, peptic ulcer, or gastrointestinal cancer was also excluded from the study. Seventeen subjects were found to be eligible and followed a diet low in phytochemicals for 5 days. CMG intake $(10 \mathrm{~g})$ took place after overnight fasting. Blood samples were collected at specific time intervals (e.g., 0, 1, 2, 4, 8, and $24 \mathrm{~h}$ ). Plasma was separated from serum by centrifugation immediately after blood collection. Finally, $1 \mathrm{~mL}$ of each sample was placed in microcentrifuge tubes and stored at $-80^{\circ} \mathrm{C}$ until further treatment.

\section{Preparation of plasma samples}

The protocol for the preparation of plasma samples was modified from Lemonakis et al. [34]. First, plasma samples were thawed in ice at $4^{\circ} \mathrm{C}$ for 30 to $60 \mathrm{~min}$. Then, $50 \mu \mathrm{L}$ of each sample was transferred in a microcentrifuge tube of $1.5 \mathrm{~mL}$ in which $2 \times 200 \mu \mathrm{L}$ of cold ACN $\left(-20^{\circ} \mathrm{C}\right)$ were added for protein precipitation. Tubes were thoroughly mixed in vortex for $15 \mathrm{~s}$ each time ACN was added to the sample. Tubes containing precipitated proteins were centrifuged at $4^{\circ} \mathrm{C}$ and $12000 \mathrm{rpm}$ for 10 min using a Mikro $200 \mathrm{R}$ centrifugator. After that, supernatants were transferred into microcentrifuge tubes and spiked with the IS in order to reach a final concentration of $40 \mathrm{ng} / \mathrm{mL}$ after reconstitution. Samples were further dried down in an Eppendorf vacufuge concentrator for $2.5 \mathrm{~h}$. No heating was applied during the drying process. Once dried, samples were stored at $-80^{\circ} \mathrm{C}$ until the day of analysis when they were reconstituted in $100 \mu \mathrm{L}$ of $\mathrm{MeOH}$.

\section{Liquid chromatography mass spectrometry of plasma samples}

LC-MS analysis was carried out using an Advance UHPLC system coupled to a Bruker EVOQ Elite Triple Quadrupole Mass Spectrometer. Separation was achieved on an Acquity HSS T3 column $(100 \mathrm{~mm} \times 2.1 \mathrm{~mm}, 1.8 \mu \mathrm{m})$ heated at $40{ }^{\circ} \mathrm{C}$ with a gradient of water containing $0.1 \%(\mathrm{v} / \mathrm{v})$ formic acid (A) and ACN (B) at a flowrate of $0.4 \mathrm{~mL} / \mathrm{min}$. Injection volume was set to $5 \mu \mathrm{L}$. Elution started at $70 \% \mathrm{~B}$ and reached $89 \% \mathrm{~B}$ in $12.5 \mathrm{~min}$, then it increased to $100 \%$ in $0.5 \mathrm{~min}$. These conditions were maintained for $3 \mathrm{~min}$ before going back to initial conditions for a 4-min reequilibration. The mass spectrometer was equipped with a heated ESI source operated in the negative mode. A tension of $5 \mathrm{kV}$ was applied to the capillary. Heated probe and cone temperatures were set at 300 and $350^{\circ} \mathrm{C}$, respectively, while probe and cone desolvation gas (nitrogen) flows were set at 40 and 20 units, respectively. The nebulizer gas flow was set at 50 units and the exhaust gas was on. Argon under a vacuum of 1.5 mTorr was used as the collision gas. Finally, acquisition was performed in pseudo-MRM mode [42]. The deprotonated molecular ion was chosen as the precursor as well as quantifier and confirmation $(453>453$ for IMNA and MNA, $497>497$ for 3-O-acetyl ursolic acid). Collision energies were set at 20 and $30 \mathrm{~V}$ for quantification and confirmation transitions, respectively. Q1 and Q3 were both operated with standard resolution settings and scan times were of 50 ms for each transition.

\section{Quality control}

Internal quality controls were systematically performed. LC-MS grade $\mathrm{MeOH}$ was used as injection blanks in order to control carryover. Moreover, procedural blanks made of high-purity water 
were prepared to monitor eventual cross contamination during sample preparation. Pooled QC samples, which consisted of thoroughly mixed small aliquots of each plasma sample to be studied, were also prepared in order to check system performance before, during, and after analytical series. Finally, blank matrix, which was prepared from small aliquots of $\mathrm{t}=0$ plasma sample from each healthy volunteer participating in the study, was spiked for the verification of method performance.

\section{Method validation}

Specificity of the LC-MS method was investigated by the analysis of standard solutions in $\mathrm{MeOH}$ of MNA and IMNA as well as TMEWP. Concentrations were $10 \mathrm{ng} / \mathrm{mL}$ of MNA and IMNA, and $50 \mu \mathrm{g} / \mathrm{mL}$ for TMEWP. The resolution between the targeted compounds was determined and retention times of each standard in relation to the TMEWP were observed.

Short-term stability tests were performed over a one week period in order to guaranty the reliability of the analytical process. Fresh standard solutions were prepared in 3 independent replicates at 3 different concentrations $(20,100,300 \mathrm{ng} / \mathrm{mL}, \mathrm{n}=9$ ) and were analyzed on 5 different days (days 1, 2, 3, 4, 5, and 7). During this period, samples were left in the autosampler at $10^{\circ} \mathrm{C}$ in similar storage conditions to those of the samples to be analyzed further.

Calibration curves were built upon 3 analytical replicates of standard solutions at 15 different levels of concentration $(\mathrm{n}=45)$. Analyte concentrations ranged from 3 to $800 \mathrm{ng} / \mathrm{mL}$ for IMNA and from 0.5 to $500 \mathrm{ng} / \mathrm{mL}$ for MNA while IS concentrations were fixed at $40 \mathrm{ng} / \mathrm{mL}$. Calibration curves were determined in intermediate precision conditions during 5 different days. Each sample was randomly injected three times. Calibration models were then analyzed by observation of $R^{2}$ and plots of residuals. Moreover, statistical evaluation of model linearity was conducted by ANOVA.

LOQs were determined by the concentration of the first level of the calibration curves. LOQs were then verified in matrix by spiking 3 sample replicates of blank matrix (pooled plasma samples $t=0$ ) accordingly. Acceptance criteria were based on the observation of the RSD of the signal response with a maximum acceptable deviation of $20 \%$ and of its difference with a standard solution at the same concentration with a maximum acceptable deviation of $30 \%$.

Method accuracy was evaluated by the measurement error of a spiked blank matrix (pooled plasma samples $\mathrm{t}=0$ ) with a maximum acceptable deviation of $20 \%$. All spiked samples were prepared in 3 independent replicates at 3 different concentrations (low, medium, and high: 20, 100, $300 \mathrm{ng} / \mathrm{mL}$, respectively, for each analyte) while the IS concentration was $40 \mathrm{ng} / \mathrm{mL}$. The experiment was repeated after one week. The SD of the measurement error between samples at each low, medium, and high levels of concentration was used to evaluate precision in intermediate precision conditions.

\section{Supporting information}

The following are available as supporting information: UPLC-HRMS profile of CMG AF with two different ionization sources (APCI/ESI) operated in the negative mode (Fig. 1S), ESI(-)-HRMS spectra of fraction 1 (IMNA) and fraction 2 (MNA) (Fig. 2S), ${ }^{1} \mathrm{H}$ NMR spectra of IMNA and MNA (Fig. 3S), calibration curves of both targeted compounds in solvent (Fig. 4S), and variances analysis of the calibration models of the targeted compounds (Table 1S).

\section{Contributors' Statement}

Concept and design of work: V.B., M.H.; data collection: E. K., P. G.; design and conduction of human trial: A.C.K., E.P.; analysis and interpretation of data: E. K., P.G., V.B., E.V.M., A.A., M.H.; drafting of manuscript: V.B., E.V.M., M.H.; statistical analysis: V. B., E. V. M.; critical revision of manuscript: A. C. K., A. A., M. H.

\section{Acknowledgements}

The present work was cofunded by the European Union (ERDF) and Greek national funds through the Operational Program "Competitiveness, Entrepreneurship and Innovation", under the call "STRENGTHENING RESEARCH AND INNOVATION INFRASTRUCTURES" (project code: 5002 803, Title: Upgrading the Plant Capital - PlantUp). Eleni V. Mikropoulou is financed through a Stavros Niarchos Foundation (SNF) grant to the National and Kapodistrian University of Athens. The authors would like to thank the Chios Mastiha Growers Association for providing the crude mastic gum.

Conflict of Interest

The authors declare that they have no conflict of interest.

\section{References}

[1] Browicz K. Pistacia lentiscus cv. Chia (Anacardiaceae) on Chios island. PI Syst Evol 1987; 155: 189-195

[2] lerapetritis DG. The geography of the Chios mastic trade from the 17th through to the 19th century. Ethnobot Res Appl 2010; 8: 153-167

[3] UNESCO. Decision of the Intergovernmental Committee: 9.COM 10.18, Inscribing the Know-how of cultivating mastic on the island of Chios on the Representative List of the Intangible Cultural Heritage of Humanity. Accessed November 25, 2020 at: https://ich.unesco.org/en/decisions/ 9.COM/10.18

[4] Pachi VK, Mikropoulou EV, Gkiouvetidis P, Siafakas K, Argyropoulou A, Angelis A, Mitakou S, Halabalaki M. Traditional uses, phytochemistry and pharmacology of Chios mastic gum (Pistacia lentiscus var. Chia, Anacardiaceae): A review. J Ethnopharmacol 2020; 254: 112485

[5] Al-Said MS, Ageel AM, Parmar NS, Tariq M. Evaluation of mastic, a crude drug obtained from Pistacia lentiscus for gastric and duodenal anti-ulcer activity. J Ethnopharmacol 1986; 15: 271-278

[6] Dabos KJ, Sfika E, Vlatta LJ, Frantzi D, Amygdalos GI, Giannikopoulos G. Is Chios mastic gum effective in the treatment of functional dyspepsia? A prospective randomised double-blind placebo controlled trial. J Ethnopharmacol 2010; 127: 205-209

[7] Magiatis P, Melliou E, Skaltsounis AL, Chinou IB, Mitaku S. Chemical composition and antimicrobial activity of the essential oils of Pistacia lentiscus var. chia. Planta Med 1999; 65: 749-752

[8] Tassou CC, Nychas GJE. Antimicrobial activity of the essential oil of mastic gum (Pistacia lentiscus var. chia) on Gram positive and Gram negative bacteria in broth and in Model Food System. Int Biodeterior Biodegrad 1995; 36: 411-420

[9] Koutsoudaki C, Krsek M, Rodger A. Chemical composition and antibacterial activity of the essential oil and the gum of Pistacia lentiscus Var. chia. J Agric Food Chem 2005; 53: 7681-7685 
[10] EMA. Assessment report on Pistacia lentiscus L., resin (mastix). Accessed November 25, 2020 at: http://www.ema.europa.eu/docs/ en_GB/document_library/Herbal_-_HMPC_assessment_report/2015/ 07/WC500190097.pdf

[11] EMA. Herbal Monograph on Pistacia lentiscus L., resin (mastix). Accessed November 20, 2020 at: https://www.ema.europa.eu/en/documents/ herbal-monograph/final-european-union-herbal-monograph-pistacialentiscus-I-resin-mastic_en.pdf

[12] Paraschos S, Magiatis P, Mitakou S, Petraki K, Kalliaropoulos A, Maragkoudakis P, Mentis A, Sgouras D, Skaltsounis AL. In vitro and in vivo activities of Chios mastic gum extracts and constituents against Helicobacter pylori. Antimicrob Agents Chemother 2007; 51: 551-559

[13] Kaliora AC, Stathopoulou MG, Triantafillidis JK, Dedoussis GVZ, Andrikopoulos NK. Chios mastic treatment of patients with active Crohn's disease. World J Gastroenterol 2007; 13: 748-753

[14] Balan KV, Demetzos C, Prince J, Dimas K, Cladaras M, Han Z, Wyche JH, Pantazis P. Induction of apoptosis in human colon cancer HCT116 cells treated with an extract of the plant product, chios mastic gum. In Vivo (Brooklyn) 2005; 19: 93-102

[15] Dimas K, Hatziantoniou S, Wyche JH, Pantazis P. A mastic gum extract induces suppression of growth of human colorectal tumor xenografts in immunodeficient mice. In Vivo (Brooklyn) 2009; 23: 63-68

[16] He M, Li A, Xu C, Wang S, Zhang M, Gu H, Yang Y, Tao H. Mechanisms of antiprostate cancer by gum mastic: NF- $\mathrm{KB}$ signal as target. Acta Pharmacol Sin 2007; 28: 446-452

[17] He ML, Chen WW, Zhang PJ, Jiang AL, Fan W, Yuan HQ, Liu WW, Zhang JY. Gum mastic increases maspin expression in prostate cancer cells. Acta Pharmacol Sin 2007; 28: 567-572

[18] Spyridopoulou K, Tiptiri-Kourpeti A, Lampri E, Fitsiou E, Vasileiadis S, Vamvakias M, Bardouki H, Goussia A, Malamou-Mitsi V, Panayiotidis MI, Galanis A, Pappa A, Chlichlia K. Dietary mastic oil extracted from Pistacia lentiscus var. chia suppresses tumor growth in experimental colon cancer models. Sci Rep 2017; 7: 3782

[19] Abdel-Rahman AHY, Youssef AM, Youssef AM. Mastich as an antioxidant. J Am Oil Chem Soc 1975; 52: 423-423

[20] Assimopoulou AN, Zlatanos SN, Papageorgiou VP. Antioxidant activity of natural resins and bioactive triterpenes in oil substrates. Food Chem 2005; 92: 721-727

[21] Andrikopoulos NK, Kaliora AC, Assimopoulou AN, Papapeorgiou VP. Biological activity of some naturally occurring resins, gums and pigments against in vitro LDL oxidation. Phytother Res 2003; 17: 501-507

[22] Loizou S, Paraschos S, Mitakou S, Chrousos GP, Lekakis I, Moutsatsou P. Chios mastic gum extract and isolated phytosterol tirucallol exhibit antiinflammatory activity in human aortic endothelial cells. Exp Biol Med 2009; 234: 553-561

[23] Dedoussis GVZ, Kaliora AC, Psarras S, Chiou A, Mylona A, Papadopoulos NG, Andrikopoulos NK. Antiatherogenic effect of Pistacia lentiscus via GSH restoration and downregulation of CD36 mRNA expression. Atherosclerosis 2004; 174: 293-303

[24] Triantafyllou A, Chaviaras N, Sergentanis TN, Protopapa E, Tsaknis J. Chios mastic gum modulates serum biochemical parameters in a human population. J Ethnopharmacol 2007; 111: 43-49

[25] Andreadou I, Mitakou S, Paraschos S, Efentakis P, Magiatis P, Kaklamanis L, Halabalaki M, Skaltsounis L, Iliodromitis EK. Pistacia lentiscus L. reduces the infarct size in normal fed anesthetized rabbits and possess antiatheromatic and hypolipidemic activity in cholesterol fed rabbits. Phytomedicine 2016; 23: 1220-1226

[26] Kartalis A, Didagelos M, Georgiadis I, Benetos G, Smyrnioudis N, Marmaras H, Voutas P, Zotika C, Garoufalis S, Andrikopoulos G. Effects of Chios mastic gum on cholesterol and glucose levels of healthy volunteers: A prospective, randomized, placebo-controlled, pilot study (CHIOS-MASTIHA). Eur J Prev Cardiol 2015; 23: 722-729

[27] González-Burgos E, Gomez-Serranillos MP. Terpene compounds in nature: a review of their potential antioxidant activity. Curr Med Chem 2012; 19: 5319-5341

[28] de Cássia da Silveira e Sá R, Andrade LN, de Sousa DP. A review on antiinflammatory activity of monoterpenes. Molecules 2013; 18: 12271254

[29] Yadav VR, Prasad S, Sung B, Kannappan R, Aggarwal BB. Targeting inflammatory pathways by triterpenoids for prevention and treatment of cancer. Toxins (Basel) 2010; 2: 2428-2466

[30] Barton DHR, Seoane E. Triterpenoids. Part XXII. The constitution and stereochemistry of masticadienonic acid. J Chem Soc 1956; 189: 41504157

[31] Assimopoulou AN, Papageorgiou VP. GC-MS analysis of penta- and tetracyclic triterpenes from resins of Pistacia species. Part I. Pistacia lentiscus var. Chia. Biomed Chromatogr 2005; 19: 285-311

[32] Marner FJ, Freyer A, Lex J. Triterpenoids from gum mastic, the resin of Pistacia lentiscus. Phytochemistry 1991; 30: 3709-3712

[33] van den Berg KJ, van der Horst J, Boon J], Sudmeijer OO. Cis-1,4-poly- $\beta$ myrcene: the structure of the polymeric fraction of mastic resin (Pistacia Lentiscus L.) elucidated. Tetrahedron Lett 1998; 39: 2645-2648

[34] Lemonakis N, Magiatis P, Kostomitsopoulos N, Skaltsounis AL, Tamvakopoulos C. Oral administration of chios mastic gum or extracts in mice: quantification of triterpenic acids by liquid chromatographytandem mass spectrometry. Planta Med 2011; 77: 1916-1923

[35] Brieudes V, Kallergis E, Papada E, Gioxari A, Kaliora A, Mitakou S, Skaltsounis L, Halabalaki M. Bioavailability study and impact of Chios mastic supplementation on oxidative stress in healthy humans. PMIO 2017; 4 (S 01): S1-S202

[36] Papada E, Gioxari A, Brieudes V, Amerikanou C, Halabalaki M, Skaltsounis AL, Smyrnioudis I, Kaliora AC. Bioavailability of terpenes and postprandial effect on human antioxidant potential. An open-label study in healthy subjects. Mol Nutr Food Res 2018; 62: 1-9

[37] ICH. Validation of analytical procedures: text and methodology, Q2(R1). Accessed September 25, 2020 at: https://www.ema.europa.eu/en/ documents/scientific-guideline/ich-q-2-r1-validation-analyticalprocedures-text-methodology-step-5_en.pdf

[38] Xynos N, Termentzi A, Fokialakis N, Skaltsounis LA, Aligiannis N. Supercritical $\mathrm{CO}_{2}$ extraction of mastic gum and chemical characterization of bioactive fractions using LC-HRMS/MS and GC-MS. J Supercrit Fluids 2018; 133: 349-356

[39] Hamzaoui M, Angelis A, Laskari V, Termentzi A, Hubert J, Fokialakis N, Aligiannis N, Renault J, Skaltsounis A. Separation of neutral and acidic triterpenes from Mastic gum using Centrifugal Partition Chromatography (CPC) and Supercritical Fluid Chromatography (SFC-CO2). Planta Med 2015; 81: PW_62

[40] Wojdyło A, Oszmiański J, Czemerys R. Antioxidant activity and phenolic compounds in 32 selected herbs. Food Chem 2007; 105: 940-949

[41] International Vocabulary of Metrology - basic and general Concepts and associated Terms (VIM, 3rd Edition). Accessed September 25, 2020 at: https://www.bipm.org/utils/common/documents/jcgm/JCGM_200_ 2012.pdf

[42] Haug LS, Thomsen C, Becher G. A sensitive method for determination of a broad range of perfluorinated compounds in serum suitable for largescale human biomonitoring. J Chromatogr A 2009; 1216: 385-393 